\title{
ARTICLE OPEN RegenDbase: a comparative database of noncoding RNA regulation of tissue regeneration circuits across multiple taxa
}

\author{
Benjamin L. King $\mathbb{D}^{1,2,3}$, Michael C. Rosenstein $\mathbb{D}^{1,5}$, Ashley M. Smith ${ }^{1}$, Christina A. Dykeman ${ }^{1}$, Grace A. Smith ${ }^{2,4}$ and Viravuth P. Yin ${ }^{1,3}$
}

Regeneration is an endogenous process of tissue repair that culminates in complete restoration of tissue and organ function. While regenerative capacity in mammals is limited to select tissues, lower vertebrates like zebrafish and salamanders are endowed with the capacity to regenerate entire limbs and most adult tissues, including heart muscle. Numerous profiling studies have been conducted using these research models in an effort to identify the genetic circuits that accompany tissue regeneration. Most of these studies, however, are confined to an individual injury model and/or research organism and focused primarily on protein encoding transcripts. Here we describe RegenDbase, a new database with the functionality to compare and contrast gene regulatory pathways within and across tissues and research models. RegenDbase combines pipelines that integrate analysis of noncoding RNAs in combination with protein encoding transcripts. We created RegenDbase with a newly generated comprehensive dataset for adult zebrafish heart regeneration combined with existing microarray and RNA-sequencing studies on multiple injured tissues. In this current release, we detail microRNA-mRNA regulatory circuits and the biological processes these interactions control during the early stages of heart regeneration. Moreover, we identify known and putative novel IncRNAs and identify their potential target genes based on proximity searches. We postulate that these candidate factors underscore robust regenerative capacity in lower vertebrates. RegenDbase provides a systems-level analysis of tissue regeneration genetic circuits across injury and animal models and addresses the growing need to understand how noncoding RNAs influence these changes in gene expression.

npj Regenerative Medicine (2018)3:10; doi:10.1038/s41536-018-0049-0

\section{INTRODUCTION}

A limited capacity to repair and regenerate injured and damaged tissues underscores many degenerative and chronic diseases. ${ }^{1-4}$ Regenerative biology seeks to define endogenous and fundamental mechanisms that can be used to stimulate human regenerative capacity. For example, drastically improved outcomes would be realized if necrotic cardiac muscle tissue regenerated after acute myocardial infarction rather than forming scar tissue that reduces cardiac output. Unlike humans, many adult vertebrates, such as ray-finned fishes and urodeles, have the capacity to regenerate many injured tissues. The zebrafish, Danio rerio, can regenerate multiple adult tissues including cardiac, ${ }^{5}$ spinal cord, ${ }^{6}$ and fin appendages. ${ }^{7}$ Urodele models of limb regeneration include the axolotl (Ambystoma mexicanum $)^{8}$ and newt (Notophtalmus viridescens). ${ }^{9}$ Studies of these individual models have advanced our understanding of regeneration genetic circuits and their regulation. Among these mechanisms are microRNAs (miRNAs), small noncoding RNAs that are essential regulators of development and adult tissue regeneration. ${ }^{10-13}$

Comparative studies of multiple models of complex tissue regeneration provide an opportunity to identify signaling pathways that are conserved or unique among models. ${ }^{14}$ Conducting these comparisons within a phylogenetic context also allows for analysis of ancestral and derived traits. These comparative studies pose challenges that existing databases have not resolved, including (1) compiling large profiling datasets on complex tissue regeneration; (2) facilitating comparisons of experiments within and across organisms; and (3) integrating noncoding RNAs as another level of regulatory control.

Current database resources used for analyzing gene expression are limited in terms of breadth of organisms, exclusion of noncoding RNAs, and a lack of focus on biological processes. The Limbform resource integrated 829 experiments to predict how temporal and spatial patterns of gene expression are associated with anatomical structures during appendage regeneration. ${ }^{15}$ While eight vertebrate species are represented in Limbform, zebrafish and mice are not among them. miRNAs, potent regulators of regeneration, are not represented in this resource. The Regeneration Gene Database (REGene) provides information about 948 genes gathered from the literature using Gene Ontology (GO) annotations and abstracts, but does not present large-scale gene expression datasets from regenerating tissues. ${ }^{16}$ Existing gene expression resources such as the Gene Expression Omnibus, ${ }^{17}$ Expression Atlas, ${ }^{18}$ or Genotype-Tissue Expression Project, ${ }^{19}$ while informative, do not specifically focus on regeneration or robustly support comparisons across datasets. Analyses of miRNA and mRNA expression data along with miRNA target predictions are well represented for 73 human datasets in miRGator. ${ }^{20}$ However, miRGator lacks the focus on tissue repair

\footnotetext{
${ }^{1}$ Kathryn Davis Center for Regenerative Biology and Medicine, Mount Desert Island Biological Laboratory, Salisbury Cove, ME 04672, USA; ${ }^{2}$ Department of Molecular and

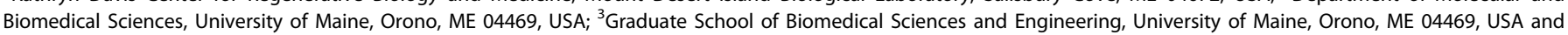
${ }^{4}$ University of Maine Honors College, University of Maine, Orono, ME 04469, USA

Correspondence: Benjamin L. King (benjamin.l.king@maine.edu) or Viravuth P. Yin (vyin@mdibl.org)

${ }^{5}$ Present address: RockStep Solutions, Portland, ME 04101, USA
}

Received: 27 November 2017 Revised: 17 April 2018 Accepted: 4 May 2018

Published online: 29 May 2018 
and regeneration. A database resource that aims to provide a systems-level analysis of tissue regeneration genetic circuits would ideally include these existing resources and provide complementary functionality pertinent to comparative studies of regeneration.

In this work, we describe a newly created Comparative Models of Regeneration Database (RegenDbase; https://regenDbase.org), which focuses on understanding the regulatory control of noncoding RNAs on evolutionarily conserved regeneration signaling pathways. Employing the unique functionality and web interface of RegenDbase, we studied newly generated zebrafish heart regeneration miRNA and mRNA expression datasets and made comparisons with similar data from neonatal mouse heart regeneration. RegenDbase was used to make predictions about stage-specific interactions between miRNAs and mRNAs during zebrafish heart regeneration. We extended our understanding of noncoding RNAs through the identification of long noncoding RNA (IncRNAs) and their putative target transcripts. We characterized a subset of six previously annotated and five novel, differentially expressed IncRNAs that were located antisense or adjacent to differentially expressed protein-coding genes. Finally, we compared early stages of zebrafish and neonatal mouse heart regeneration to identify orthologous genes that were commonly differentially expressed. The integrated functionality within RegenDbase has the potential to advance our understanding of tissue regeneration by accelerating the identification of candidate genes for functional studies through a cross-species comparative strategy.

\section{RESULTS}

Comparative Models of Regeneration Database

The Comparative Models of Regeneration Database (RegenDbase) is a resource designed to identify and compare signaling pathways that underlie tissue regeneration across research models (Fig. 1). RegenDbase is an integrated, multi-faceted platform consisting of data analysis pipelines, a relational database, database load software, and web interface software. Represented within RegenDbase are genes, transcripts, and miRNAs from Ensembl $^{21}$ and miRBase ${ }^{22}$ for zebrafish, axolotl, and mouse. In order to integrate additional studies in the future, we also represent human and C. elegans genes, transcripts and miRNAs. $\mathrm{GO}^{23}$ functional annotations, pathway assignment from BioSystems, ${ }^{24}$ and homology relationships among genes from OrthoDB ${ }^{25}$ are also integrated together in the RegenDbase platform (Fig. 1a; Supplementary Fig. S1).

Integration of published and unpublished data derived from several disparate sources required defined rules to clean and validate data prior to mapping relationships. Gene and miRNA expression data, currently from zebrafish, axolotl, and mouse (Supplementary Table S1), were reanalyzed using consistent microarray or RNA-Seq analysis workflows to incorporate up-todate gene annotation. Analysis results were represented as pairwise contrasts between pairs of sample groups and can be filtered by several different strategies including significance, direction of fold change, GO annotations, and BioSystems pathway membership.

A key feature of RegenDbase is the methodology to define the regulatory control of noncoding RNAs during regeneration. First, RegenDbase enables investigation of how miRNAs regulate differentially expressed genes in two ways. RegenDbase generates miRNA target predictions for human, mouse, zebrafish, and $C$. elegans using up-to-date Ensembl annotation of $3 p$ UTR sequences. Using these miRNA target predictions, differentially expressed genes may be filtered by whether they are predicted targets of any miRNAs specified in a list (Fig. 1a). Alternatively, differentially expressed genes may be identified that are co- targeted by up to three miRNAs. Second, RegenDbase can identify differentially expressed IncRNAs and associated neighboring genes along the chromosome that represent putative IncRNA target genes. Differentially expressed genes may be filtered by proximity to identify gene pairs that are adjacent to one another within a window size up to 10 genes. These gene pairs may represent potential cases where an IncRNA could regulate a protein-coding gene ${ }^{26}$ or gene clusters. ${ }^{27}$

RegenDbase provides a unique approach for comparing gene and miRNA expression data. Filtered subsets of differentially expressed mRNAs or miRNAs from two pairwise contrasts may be compared to identify shared or unique genes (Fig. 1b, c). These comparisons may be applied within and across experiments and organisms. For instance, comparative analyses from a single experiment can be used to survey different stages of regeneration and identify candidate markers unique to a stage, or common across multiple stages. By comparing uninjured and 3 days postamputation (dpa) time points of heart regeneration, we can readily identify known regeneration genes, such as the chemokine (C-X-C motif) receptor $4 \mathrm{~b}, c x c r 4 b,{ }^{28}$ or novel candidate genes for functional studies (Fig. 1c). RegenDbase has the functionality to rapidly display the expression changes for $c x c r 4 b$ or any other gene across all stages of heart regeneration (Fig. 1c). Alternatively, intra-species comparisons may be performed to identify shared genes for regeneration following different injury models. For example, the zebrafish heart ventricular resection injury may be compared to the cryoinjury model. Alternatively, the expression of proliferative genes during blastema formation during regeneration of zebrafish caudal fins could be compared to early stages of zebrafish heart regeneration. Integration of homology data into RegenDbase permits searches of orthologous genes across regeneration datasets. This functionality allows for comparisons across species, such as comparing zebrafish and neonatal mouse heart regeneration (Fig. 1a).

Integrating a new dataset for zebrafish heart regeneration To better understand the transcriptional changes in gene expression during zebrafish heart regeneration, we profiled miRNA and mRNA expression across a time course of zebrafish heart regeneration, from $0,1,3,7,14,21$ to $30 \mathrm{dpa}$. These time points encompass the various cellular processes of heart regeneration that are coordinated to ensure synthesis of new heart tissue and restoration of tissue function. ${ }^{29}$ These stages include clotting and endocardial activation (1 dpa), epicardial activation and cardiomyocyte dedifferentiation and proliferation (3 dpa), peak cardiomyocyte proliferation (7 dpa), vascularization and muscle integration with the uninjured heart tissue (14 dpa), electrical coupling and clot dissolution (21 dpa), and functional recovery of tissue function ( $30 \mathrm{dpa})$. We compared the uninjured ( $0 \mathrm{dpa}$ ) samples to each of the subsequent stages and integrated these pairwise contrasts into RegenDbase using both noncoding and protein encoding transcripts.

\section{Stage-specific miRNA expression profiles of zebrafish heart} regeneration

miRNAs are critical regulators of gene expression during development and regeneration. ${ }^{10,11,13,14,30,31}$ However, a comprehensive atlas of miRNA expression throughout heart regeneration has not been previously reported. Our analyses of zebrafish heart regeneration identified a total of 103 mature miRNAs that were differentially expressed between $0 \mathrm{dpa}$ and at least one stage during heart regeneration (Fig. 2a; Supplementary Table S2). A total of $38(37 \%)$ miRNAs were differentially expressed at a single time point including 18 at $1 \mathrm{dpa}, 12$ at $3 \mathrm{dpa}, 1$ (Mir-199-P1_5p*) at $7 \mathrm{dpa}, 6$ at $21 \mathrm{dpa}$, and 1 (MiR-425_5p) at $30 \mathrm{dpa}$. Only Mir-135-P3a and Mir-146-P2, an miRNA upregulated in several cancers, ${ }^{32}$ were consistently upregulated across all time points. This general 
a

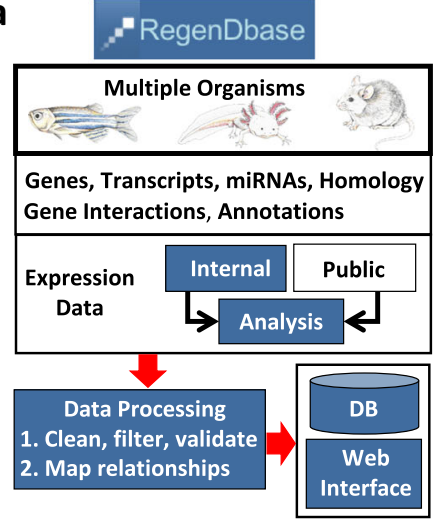

b

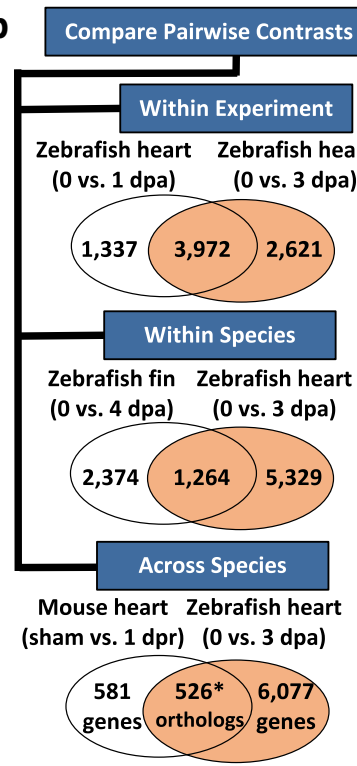

C

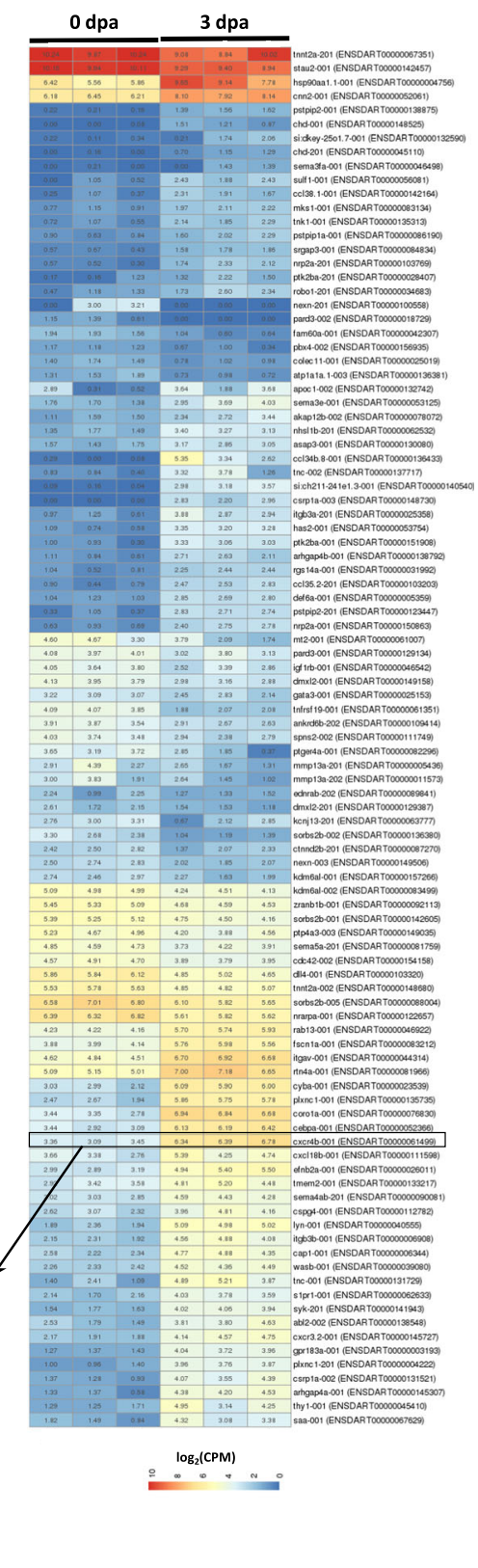

Fig. 1 RegenDbase provides fundamental analyses of regeneration circuits within individual experiments and comparative analyses across experiments and species. a Organizational workflow for multi-layered data processing and analysis of published (public) and unpublished (internal) gene expression datasets. b Examples of how RegenDbase was used to compare: (i) genes common or unique to two pairwise contrasts of early stages of zebrafish heart regeneration ( 0 vs. 1 dpa and 0 vs. $3 \mathrm{dpa}$ ); (ii) genes common or unique to contrasts from early stages of zebrafish caudal fin regeneration ( 0 vs. $4 \mathrm{dpa}$ ) and zebrafish heart regeneration ( 0 vs. 3 dpa); (iii) comparative analyses of early zebrafish heart ( 0 vs. $3 \mathrm{dpa}$ ) and neonatal mouse heart ( $0 \mathrm{vs} .1 \mathrm{dpa}$ ) regeneration showing the number of orthologs commonly differentially expressed along with the number of zebrafish and mouse genes unique to each model. $c$ Heatmap representing genes from precomputed pairwise contrasts from a subset of significantly up- and downregulated transcripts between uninjured and 3 days post-amputation (dpa) regenerating adult ventricles. The temporal expression profile for $c x c r 4 b$, an essential regeneration gene, is shown as a boxplot in the web interface

pattern of unique and common miRNAs suggests that there are stage-specific and overlapping regulatory networks.

Previous regeneration studies from our group and others have shown that the magnitude and volume of gene expression changes are most robust at early time points following tissue injury. ${ }^{10,11,14,33}$ Consistent with these observations, we noted that 1 and 3 dpa time points had more differentially expressed mature miRNAs compared to $0 \mathrm{dpa}$ than later time points. Thirty-six
miRNAs were commonly differentially expressed at both 1 and $3 \mathrm{dpa}$ with 35 miRNAs unique to $1 \mathrm{dpa}$ and 22 unique to $3 \mathrm{dpa}$. The known regulator of zebrafish heart regeneration, Mir-133P3a_3p, ${ }^{12}$ was one of 36 commonly downregulated miRNAs at 1 and 3 dpa (Fig. 2b). During peak cardiomyocyte proliferation at $7 \mathrm{dpa}, 23$ of the 26 differentially expressed miRNAs are conserved with humans and associated with the regulation of cellular proliferation and cancer, such as Mir-21-P1_5p (ref. ${ }^{34}$ ) (Fig. 2b). 
a

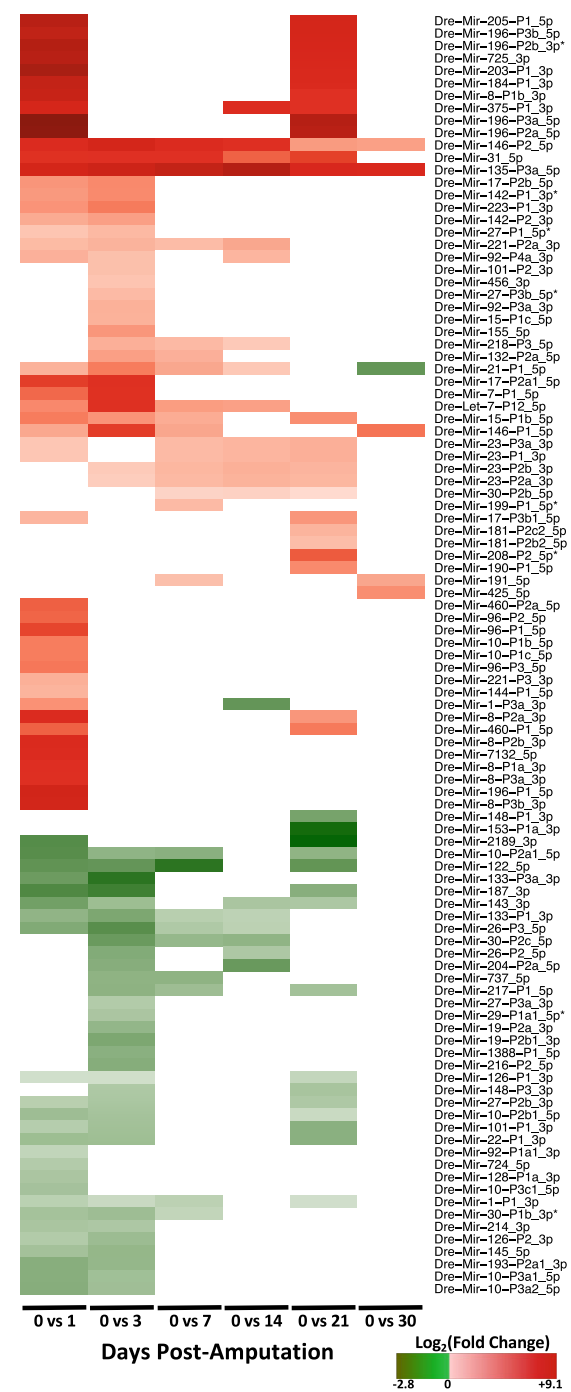

b

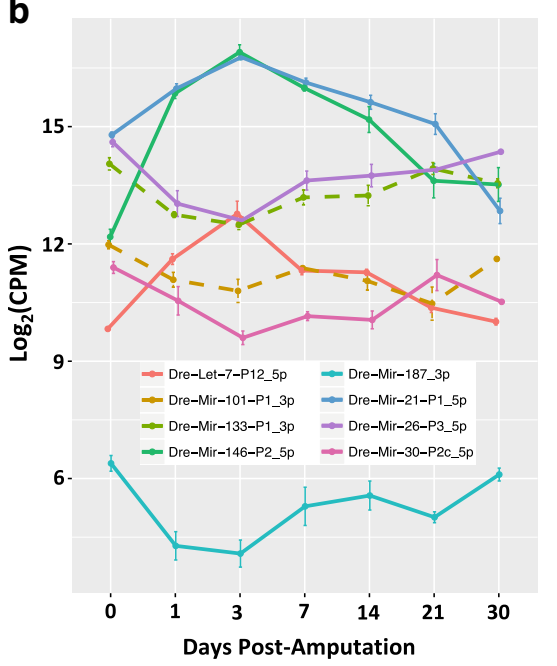

Fig. 2 miRNAs are differentially regulated during zebrafish heart regeneration. a Heatmap of differentially expressed miRNAs for each pairwise comparison between uninjured $(0 \mathrm{dpa})$ and subsequent time points. Mature miRNA nomenclature is from miRGeneDB. $\mathbf{b}$ Expression profile of miRNAs previously demonstrated to be required for zebrafish heart regeneration (Dre-Mir-133-P1_3p and Dre-Mir-101-P1_3p), miRNAs that were upregulated (Dre-Mir-21-P1_5p, Dre-Mir-146-P2_5p and Dre-Let-7-P12_5p), and miRNAs that were downregulated (Dre-Mir26-P3_5p, Dre-Mir-30-P2c_5p, and Dre-Mir-187_3p) during early stages (1 and $3 \mathrm{dpa}$ ) of heart regeneration. Error bars indicate standard error of the mean

Eight of the downregulated miRNAs conserved with humans (Mir1-P1_3p, Mir-10-P2a1_5p, Mir-26-P3_5p, Mir-30-P1b_3p, Mir-30P2c_5p, Mir-122_5p, Mir-133-P1_3p, Mir-217-P1_5p) were tumor suppressors downregulated in cancer. ${ }^{35,36}$ Of the 17 upregulated miRNAs, 9 (Mir-21-P1_5p, Mir-23-P1_3p, Mir-23-P3a_3p, Mir-30P2b_5p, Mir-31_5p, Mir-132-P2a_5p, Mir-135-P3a_5p, Mir-191_5p, Mir-221-P2a_3p) have been positively associated with cancer, but 8 (Let-7-P12_5p, Mir-15-P1b_5p, Mir-23-P2a_3p, Mir-23-P2b_3p, Mir-146-P1_5p, Mir-146-P2_5p, Mir-199-P1_5p*, Mir-218-P3_5p) have been described as tumor suppressors. ${ }^{35-37}$ The 21 dpa time point had the third largest set of differentially expressed miRNAs, and these overlapped more with $1 \mathrm{dpa}$ (30 miRNAs) and $3 \mathrm{dpa}$ (18 miRNAs) than later time points of $14 \mathrm{dpa}$ (10 miRNAs) and $30 \mathrm{dpa}$ (2 miRNAs). Furthermore, the common subset of miRNAs shared between 1 and $3 \mathrm{dpa}$ were consistently up- (16 miRNAs) or downregulated (20 miRNAs). Among the downregulated miRNAs at 1,3 , and $21 \mathrm{dpa}$ was the known regulator of zebrafish heart regeneration, Mir-101-P1_3p (ref. ${ }^{13}$ ) (Fig. 2b). The stage-specific modulations in miRNA expression suggest that subsets of miRNAs regulate distinct cellular processes during heart regeneration.
mRNA expression profiling of zebrafish heart regeneration Comprehensive mRNA expression profiling studies provide an unbiased and invaluable gene catalog for defining the genetic circuits that orchestrate heart regeneration. Consistent with the miRNA expression profiles, subsets of genes were temporally activated and repressed, as shown by sorting genes by the average level of normalized expression across samples at each time point (Supplementary Fig. S2, Supplementary Table S3). Pairwise contrasts between each injury time point with uninjured tissues showed that early stages of regeneration ( 1 and $3 \mathrm{dpa}$ ) had the largest number of differentially expressed genes (Supplementary Table S4). One dpa had 3005 genes and 3 dpa had 3533 genes with 2225 genes in common. Comparisons with subsequent time points had fewer differentially expressed genes: 1178 at $7 \mathrm{dpa}$, 1065 at $14 \mathrm{dpa}, 653$ at $21 \mathrm{dpa}$, and 882 at $30 \mathrm{dpa}$.

These differentially expressed genes were assigned to annotated cellular processes and shown as a percentage of genes associated with metabolism, heart development, immune function, cell migration, and cell proliferation (Supplementary Fig. S2). These diverse cellular responses illustrate the dynamics of 
biological processes during regeneration. The proportion of immune, cell migration, and cell proliferation genes was relatively constant. However, the proportion of metabolism, development, and heart development genes had large changes. For metabolism genes, both the smallest proportion of upregulated and largest proportion of downregulated genes were found at $7 \mathrm{dpa}$. The increase in proportions of developmental process and heart development genes peaks at $21 \mathrm{dpa}$ before returning to baseline. Looking across all pairwise-stage comparisons, we identified 11 genes that were consistently upregulated and 103 that were consistently downregulated (Supplementary Table S5). Two examples of consistently upregulated genes include the suppressor of cytokine signaling $3 a$ ( $\operatorname{socs} 3 a$ ) and myelocytomatosis oncogene $\mathrm{b}(m y c b)$.

Our analyses identified 43 genes that were differentially expressed and previously shown to be associated with zebrafish heart regeneration (Supplementary Table S6). These include upregulation of retinoic acid (RA) signaling and the RA synthesis enzyme, aldehyde dehydrogenase 1 family, member A2 (aldh1a2). The RA signaling cascade is rapidly activated in the endocardium and epicardium to create an instructive microenvironment for cardiomyocyte proliferation. ${ }^{38}$ Fibronectin $1 \mathrm{a}$ and $1 \mathrm{~b}$ (fn $1 \mathrm{a}$ and $f n 1 b)$, Wilms tumor $1 \mathrm{~b}(w t 1 b)$, and the tcf 21 and $t b x 18$ transcription factors were highly upregulated by 1 and $3 \mathrm{dpa}$, consistent with essential roles in epicardial activation. ${ }^{39-41}$ Cardiomyocyte migration requires expression of the chemokine, $c x c / 12 b$, and its receptor, $c x c r 4 b{ }^{28}$ Both transcripts were upregulated during early stages of regeneration (Fig. 1c). Two of the genes, cx43 and myl7, have been associated with heart regeneration. $c x 43$ is required for heart regeneration and has been shown to be regulated by miR$133 a .^{12}$ The cardiomyocyte myosin light chain 7 gene, $m y / 7,{ }^{42}$ is a commonly used marker to identify differentiated cardiomyocytes.

In addition to the identification of known heart regeneration genes, we identified promising new candidate genes that were also differentially expressed and had known association with heart development. Within this group of 114 genes, we identified a subset of 19 genes that also had known roles in cellular migration (Supplementary Table S5). The RAS oncogene member, rap1b, a small GTPase that functions with fibronectin $(f n 1)$ to regulate integrin signaling, ${ }^{43}$ was highly upregulated at 1,3 , and $14 \mathrm{dpa}$. Zebrafish rap $1 b$ morphants displayed myocardium defects and impaired heart contractility. ${ }^{44}$ Similarly, the cysteine and glycine rich protein 1a, csrp1a, was upregulated at 1, 3, 7, and $14 \mathrm{dpa}$. csrp1a regulates mesoderm cell migration through the noncanonical Wnt pathway during heart development. ${ }^{45}$ Given that depletion of rap $1 b$ and csrp $1 a$ activity culminates in heart defects and embryonic lethality, ${ }^{44,45}$ defining potential roles during heart regeneration will require inducible strategies to deplete gene function in the adult animal.

Our analyses of the 114 differentially expressed genes also identified natriuretic peptide receptor, npr3, and Semaphorin factor, sema3d, genes with roles in heart development and cellular proliferation (Supplementary Table S7). The natriuretic peptide receptor, npr3, regulates cardiomyocyte proliferation under conditions of low levels of natriuretic peptides, nppa and $n p p b .^{46}$ Semaphorins are secreted factors that have been shown to have numerous roles in development of the nervous system as well as the neural crest. Morpholino knockdown of sema3d during embryonic development showed defective migration of neural crest cells to the primary heart field prior to heart tube formation. ${ }^{47}$ Lack of cell migration resulted in $20 \%$ fewer cardiomyocytes in the primary heart field. Based on their expression profiles and known function in heart development, $n p r 3$ and sema3d are strong candidate genes for functional studies in heart regeneration.

Two genes demonstrate the dynamics of gene expression during heart regeneration as they were upregulated at early time points and then downregulated later, or vice versa. The extracellular matrix gene, transgelin (tagln), is a highly conserved gene expressed in smooth muscle as well as skeletal and cardiac muscle during mouse development. ${ }^{48}$ Tagln was upregulated at 1, 3,7 , and $14 \mathrm{dpa}$, and then downregulated at 21 and $30 \mathrm{dpa}$ (Supplementary Fig. S3). The transducer of ERBB2 (tob1a) is required for dorsoventral patterning and inhibits proliferation by blocking $\beta$-catenin/LEF-1 transcriptional complexes. ${ }^{49}$ Consistent with its negative regulation of proliferation, tob1a was downregulated by 2.04 -fold at $1 \mathrm{dpa}$ and 2.71 -fold at $3 \mathrm{dpa}$ and then upregulated by 1.82 -fold at $21 \mathrm{dpa}$ (Supplementary Fig. S3). These two genes represent novel candidates for heart regeneration based on their expression patterns and documented roles during development.

Stage-specific clustering of biological processes during zebrafish heart regeneration

To better understand the dynamic regulation of diverse biological processes during heart regeneration, we computed enriched GO Biological Process terms for each stage using GOrilla. ${ }^{50}$ Across all stages, a total of 185 unique Biological Process terms were enriched. Like the pattern of differentially expressed miRNAs, Fig. 3 shows sets of enriched GO terms that specifically define each stage of regeneration. One hundred and nine processes define $1 \mathrm{dpa}, 112$ for $3 \mathrm{dpa}, 43$ for $7 \mathrm{dpa}, 18$ for $14 \mathrm{dpa}, 11$ for $21 \mathrm{dpa}$, and 30 for $30 \mathrm{dpa}$ (Supplementary Table S8). The top 10 enriched GO terms at $3 \mathrm{dpa}$ are listed in Fig. 3b. Interestingly, the vast majority of these biological processes are annotated to metabolic processes. A subset of GO processes at an individual stage overlapped with neighboring stages, with 73 enriched processes shared between 1 and $3 \mathrm{dpa}$, 39 between 3 and $7 \mathrm{dpa}, 2$ between 7 and $14 \mathrm{dpa}$, and 2 between 14 and $21 \mathrm{dpa}$. There was no overlap between 21 and $30 \mathrm{dpa}$. The overlap of $73 \mathrm{GO}$ terms between 1 and 3 dpa have parental terms including Nucleotide Biosynthesis, Response to Stimulus, Protein Folding, Actin Cytoskeleton Organization, and Generation of Precursor Metabolites and Energy.

The Immune System Process GO term was enriched at 1 and $7 \mathrm{dpa}$. At the individual gene level, a total of 211 Immune System Process genes were differentially expressed for at least one of the time points. Approximately $18 \%$ of the 211 Immune-Related Genes were consistently differentially expressed at 1, 3, and $7 \mathrm{dpa}$. Epor, clpxa, slc11a2, and zgc:101846 were consistently downregulated across all time points, and epor, ${ }^{51}$ clpxa $^{52}$ and slc $11 a 2^{53}$ have known roles in hematopoiesis or erythropoiesis. How and to what extent these immune genes influence tissue regeneration is less understood.

Although tissue regeneration is generally regarded as a continuum of multiple cellular processes that coordinate the replacement of dying tissue with functional cells, we noted that 88 $(47 \%)$ of the 185 enriched processes were specifically enriched at a single time point, suggesting that these processes align with distinct stages. The time point with the most stage-specific terms was $1 \mathrm{dpa}$, with 31 unique enriched processes. As a percentage, 14,21 , and $30 \mathrm{dpa}$ had the highest proportions of stage-specific terms, 39\% (7/18), 45\% (5/11), and 77\% (23/30), respectively. For example, Negative Regulation of Cell Fate Commitment (GO:0010454) was enriched at $14 \mathrm{dpa}(p=0.00085)$ with four differentially expressed genes, fgf17, klf2a, klf2b, and tmem88a (Supplemental Table S8). Both Krupple-like transcription factors, klf $2 a$ and $k l f 2 b$, were upregulated at 14 and $21 \mathrm{dpa}$, and are associated with pluripotency and self-renewal of mammalian embryonic stem cells. ${ }^{54}$ Studies of zebrafish development have demonstrated essential roles for $k l f 2 a$ and $k l f 2 b$ in ectoderm development. $^{55}$ 
miRNA control of regenerative processes

After defining a high-level map of enriched Biological Process terms for each stage, we next asked which miRNAs regulate biological processes involved with early events in heart regeneration. Cardiomyocyte proliferation and migration are required for regeneration; these coupled processes begin by $1 \mathrm{dpa}$ and peak between 3 and 7 dpa. $^{56}$ Using expression data from $3 \mathrm{dpa}$, we identified the subset of predicted target genes for the 26 significantly upregulated and 32 downregulated miRNAs that were annotated to any of the 112 enriched GO Biological Process terms (Fig. 3c). As miRNA binding to target gene can result in mRNA degradation, ${ }^{57}$ we required that target genes have inverse expression compared to the miRNA. For upregulated miRNAs,

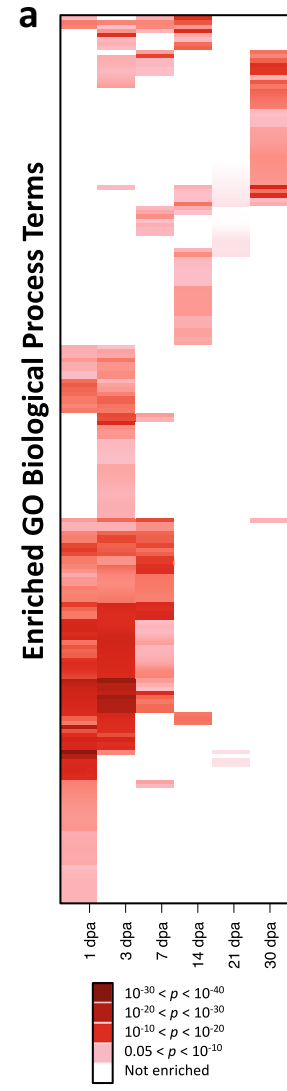

C

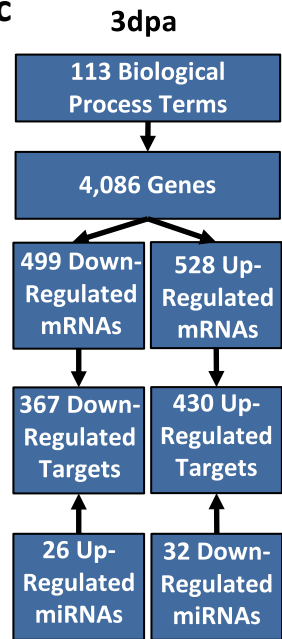

\begin{tabular}{|l|c|c|}
\hline \multicolumn{1}{|c|}{ Top 10 Enriched GO Biological Process Terms } & \# Genes & P-value \\
\hline small molecule metabolic process (GO:0044281) & 200 & $4.19 \mathrm{E}-07$ \\
\hline nucleoside monophosphate metabolic process (GO:0009123) & 50 & $3.68 \mathrm{E}-07$ \\
\hline ribonucleoside monophosphate metabolic process (GO:0009161) & 48 & $1.33 \mathrm{E}-06$ \\
\hline purine ribonucleoside monophosphate metabolic process (GO:0009167) & 44 & $8.39 \mathrm{E}-07$ \\
\hline nucleotide metabolic process (GO:0009117) & 71 & $1.34 \mathrm{E}-06$ \\
\hline oxidation-reduction process (GO:0055114) & 159 & $3.00 \mathrm{E}-06$ \\
\hline nucleobase-containing small molecule metabolic process (GO:0055086) & 80 & $4.52 \mathrm{E}-06$ \\
\hline nucleoside triphosphate metabolic process (GO:0009141) & 41 & $9.37 \mathrm{E}-06$ \\
\hline generation of precursor metabolites and energy (GO:0006091) & 44 & $9.48 \mathrm{E}-06$ \\
\hline single-organism process (GO:0044699) & 1171 & $1.56 \mathrm{E}-05$ \\
\hline
\end{tabular}

d

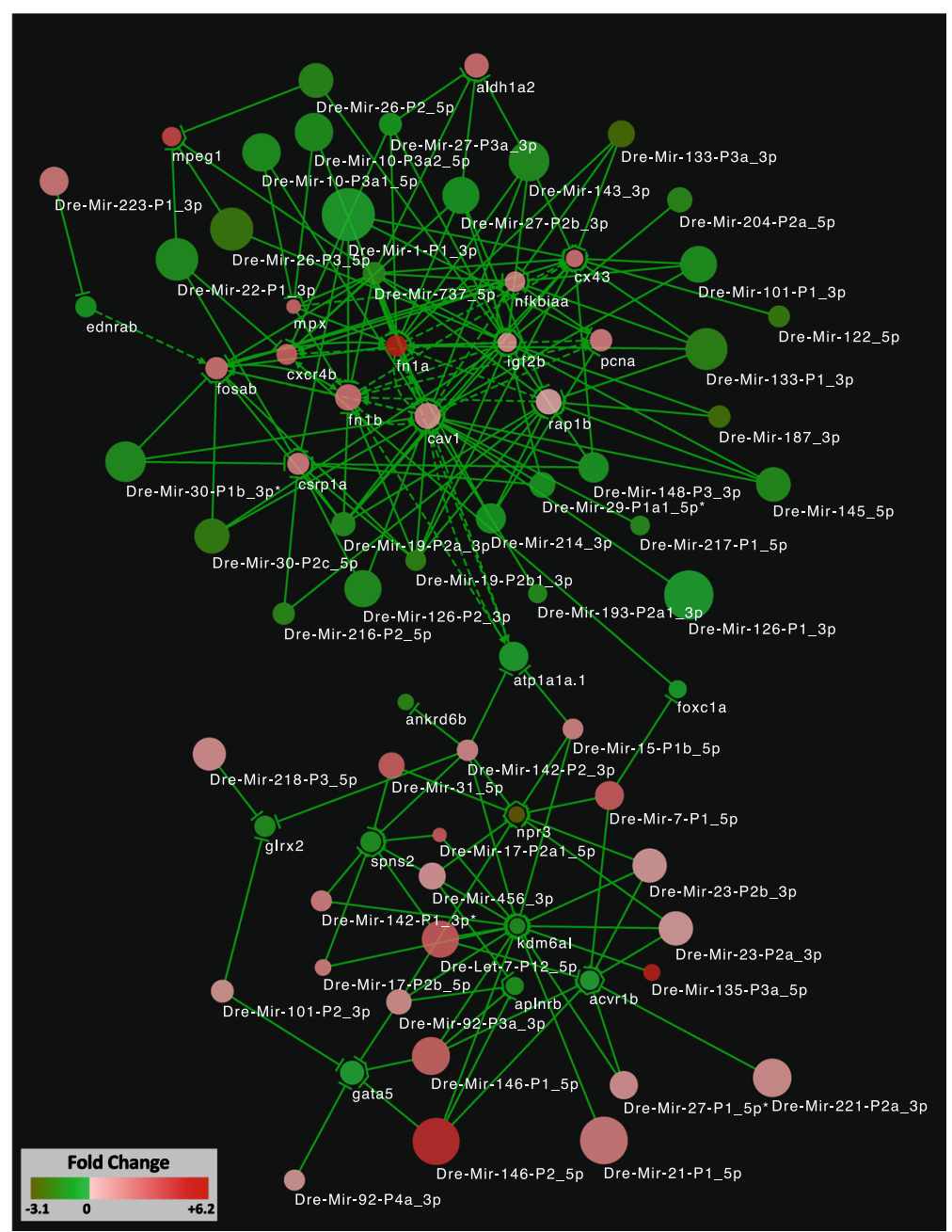

Fig. 3 Regulation of enriched Gene Ontology (GO) biological process terms by miRNAs. a Patterns of enriched GO terms for differentially expressed mRNAs at 1, 3, 7, 14, 21, and $30 \mathrm{dpa}$ relative to $0 \mathrm{dpa}$, as shown by two-dimensional hierarchical clustering. Enriched terms are colored from white (not enriched) to red (enriched) based on $\log _{2}$ (FDR). b Top 10 enriched GO terms for genes differentially expressed at $3 \mathrm{dpa}$ relative to $0 \mathrm{dpa}$. c Workflow to identify set of mRNAs differentially expressed at 3 dpa relative to $0 \mathrm{dpa}$ predicted to be targeted by miRNAs that were also differentially expressed at 3 dpa relative to $0 \mathrm{dpa}$. $\mathbf{d}$ String analysis depicting a subset of miRNA-mRNA network for 3 dpa relative to 0 dpa containing 22 upregulated and 29 downregulated miRNAs predicted to regulate 14 upregulated and 11 downregulated mRNAs known to be required for zebrafish heart regeneration 
there were 367 downregulated predicted targets and 430 upregulated predicted targets of the downregulated miRNAs. Among these targets were 14 upregulated and 11 downregulated genes previously identified to be required for heart regeneration and/or development (Fig. 3d). A general feature of this network is that several miRNAs are predicted to regulate the same target genes, suggesting synergistic downregulation of the mRNA. This network includes the known miRNA targeting interaction between the downregulated miRNA, Mir-133-P3a_3p, and the upregulated target cell junction gene, connexin $43(c x 43)^{12}$ (Fig. 3d).

Recently, our group demonstrated that miR-101 depletion was required for zebrafish heart regeneration by stimulating expression of the transcription factor and miR-101 target gene, fos. ${ }^{13}$ To further explore potential roles in which miR-101 may regulate transcription factors, we used RegenDbase to identify all transcriptional factors that were upregulated at $3 \mathrm{dpa}$ and predicted to be targeted by miR-101. Six transcription factors were identified, including two members of the FOS family, fos and fos/2 (Supplementary Table S9). The macrophage and B-cell transcription factors, spila and spi1b, are both upregulated. The C/EBP transcription factor, cebpb, has a required role in epicardial activation during zebrafish heart development and following injury. ${ }^{58}$ This analysis shows the utility of RegenDbase to identify both known and new candidate genes through the integration of miRNA targeting information. Although we did not consider multiple miRNAs in this analysis, the RegenDbase miRNA search function can be used to identify genes co-targeted by two or three miRNAs. Thus, RegenDbase is a powerful tool to elucidate miRNA regulatory networks in regeneration.

\section{IncRNA expression during zebrafish heart regeneration}

IncRNAs are multi-exonic transcripts >200nt without known protein function that control gene expression at the epigenetic, transcription and post-transcriptional levels. ${ }^{59,60}$ Recently, IncRNAs have been shown to control key genetic circuits important for development, disease, and tissue repair. ${ }^{61-66}$ The identification and extent that IncRNAs control cardiac regeneration circuits, however, remain largely unknown. Employing the RegenDbase functionality for analyses of RNA-sequencing dataset across zebrafish heart regeneration, we identified a subset of previously annotated IncRNAs that were differentially expressed during 1 and $3 \mathrm{dpa}$ of regeneration and the antisense or adjacent proteincoding genes that were also differentially expressed (Supplementary Fig. S1)

Differentially expressed IncRNAs were first identified by annotated biotype. As some IncRNAs have been shown to regulate the expression of neighboring protein-coding genes, we used the RegenDbase proximity search features to identify the subset of IncRNAs that have overlapping gene coordinates or that were adjacent to a differentially expressed protein-coding gene. Lnc-si:ch211-169j21.5 transcript is antisense to $\operatorname{arcp5b}$, encoding a tumor suppressor, and adjacent to the extracellular matrix gene, hemicentin 1 (hmcn1). Expression of $h m c n 1$ is inversely correlated with Inc-si:ch211-169j21.5 at 1 and 3 dpa (Fig. 4a). Mouse hmcn 1 has been shown to have a role in myocardium remodeling in a mouse model of myocardial infarction. ${ }^{67}$ Lnc-si:ch211-260022.1 also had an opposite expression pattern to the centrosomal protein 85 like (cep85/) gene. Translocations involving human CEB85L have been found in patients with myeloproliferative neoplasms. ${ }^{68}$ The Inc-si:ch211-260n1.5 spans several exons of the foxp2 transcription factor on the opposite strand. Mouse foxp2 mutants have abnormal electrical conduction systems, displaying R-wave amplitudes. ${ }^{69}$ The growth factor periostin (postnb) was upregulated and the neighboring Inc-si:dkey-74i1.3 was downregulated. The Inc-si:ch211-108p6.6 had opposite expression to zgc:198241, a gene that encodes a protein with an immunoglobulin domain. A regulator for cell cycle progression, $h p 1 b p 3,{ }^{70}$ was also upregulated and its neighboring Inc-si:dkey-61018.2 was downregulated. The expression changes for IncRNAs and potential target transcripts were validated using $\mathrm{qPCR}$ analysis from uninjured, 1, and 3 dpa regenerating heart tissues (Fig. 4a). Expression by qPCR correlated with the RNA-Sequencing results for all IncRNAs except for Inc-si:ch211-262n1.5 and Inc-si:dkey-74i1.3 at $1 \mathrm{dpa}$. The expression patterns for the protein-coding genes were also confirmed by qPCR except for hmcn1, ceb85l, foxp2 at $1 \mathrm{dpa}$ and $h p 1 b p 3$ (Fig. 4a).

Next, we examined the cellular distribution of these six IncRNAs and neighboring protein-coding genes at $3 \mathrm{dpa}$ within the three major cardiac tissues: epicardium, myocardium, and endocardium. We resected adult zebrafish hearts, dissociated ventricles, and isolated cells using fluorescence-activated cell sorting (FACS) (Fig. $4 b, c)$. Total RNA was subsequently extracted for qPCR studies. Interestingly, these studies showed that most IncRNAs and their putative target mRNAs are enriched in the epicardium and endocardium, two tissues that craft an instructive microenvironment for new cardiomyocyte synthesis (Fig. 4c). We observed strong expression of arpc5b, hmcn1, zgc:198241, foxp2, postnb, and cep85/ in the epicardium and endocardium. The three IncRNAs antisense or adjacent to $\operatorname{arpc5b}, h m c n 1$, foxp2, and cep85/ were also strongly expressed in the epicardium and endocardium. The expression of Inc-si:dkey-74/1.3 was highest in cardiomyocytes and lowest in the epicardium, a profile that is opposite to the expression distribution of postnb. The inverse expression patterns suggest that Inc-si:dkey-74/1.3 may negatively regulate postnb expression in the epicardium and cardiomyocytes. Both genes were expressed at approximately equal levels in the endocardium. The expression of Inc-si:ch211-108p6.6 was not detected in the three major cardiac tissues. Quantification of $\mathrm{cm} / \mathrm{c} 2, \mathrm{tcf} 21$, and fli1 expression levels in sorted cells confirm the enrichment of cardiac differentiation markers in specific cell populations after FACS (Fig. $4 d$ ). Thus, the expression and distribution analyses of IncRNAs suggest that these noncoding RNAs regulate myocardium regeneration by stimulating epicardium and endocardium activity to create an instructive injury microenvironment.

Identification of novel IncRNAs expressed during early stages of zebrafish heart regeneration

We applied an analysis workflow to identify potential novel IncRNAs expressed during heart regeneration that had not been previously annotated (Supplementary Fig. S4a). After modeling transcripts based on aligned reads using StringTie, ${ }^{71}$ we identified a set of 3741 genes not annotated by Ensembl and identified a subset of 2820 putative noncoding genes. A set of 743 putative novel IncRNAs had multiple exons and a length greater than $200 \mathrm{bp}$ and 429 were differentially expressed at early ( 1 and $3 \mathrm{dpa}$ ) or later time points compared to uninjured tissues (Supplementary Fig. S4b, Supplementary Table S10 and S11). Examination of the 119 putative novel IncRNAs unique to $1 \mathrm{dpa}$ and/or $3 \mathrm{dpa}$ revealed 28 that were not highly repetitive and lacked strong protein alignments. Five of these candidates were antisense or adjacent to differentially expressed protein-coding genes with previously described roles in cell proliferation or heart development (Fig. 5 ). A cell growth regulator, sulfatase $2 b$ (sulf $2 b$ ), that regulates WNT signaling ${ }^{72}$ was proximal to a possible novel IncRNA, Incsulf $2 b$, and antisense to another potential novel IncRNA, Inc-sulf2b$a s$. Expression of sulf $2 b$ was increased during both heart regeneration as well as a previous study of zebrafish caudal fin regeneration. $^{73}$ The putative novel IncRNA, Inc-dusp6, was proximal to dual specificity phosphatase 6 (dusp6), and both genes were downregulated during early time points. Dusp6 mutant mice had increased numbers of cardiomyocytes and heart weight. $^{74}$ Lnc-susd2 was proximal to sushi domain containing 2 (susd2), a receptor shown to interact with galectin-1 to inhibit cellular proliferation in studies of the human homolog. ${ }^{75}$ Lnc- $^{-}$ 

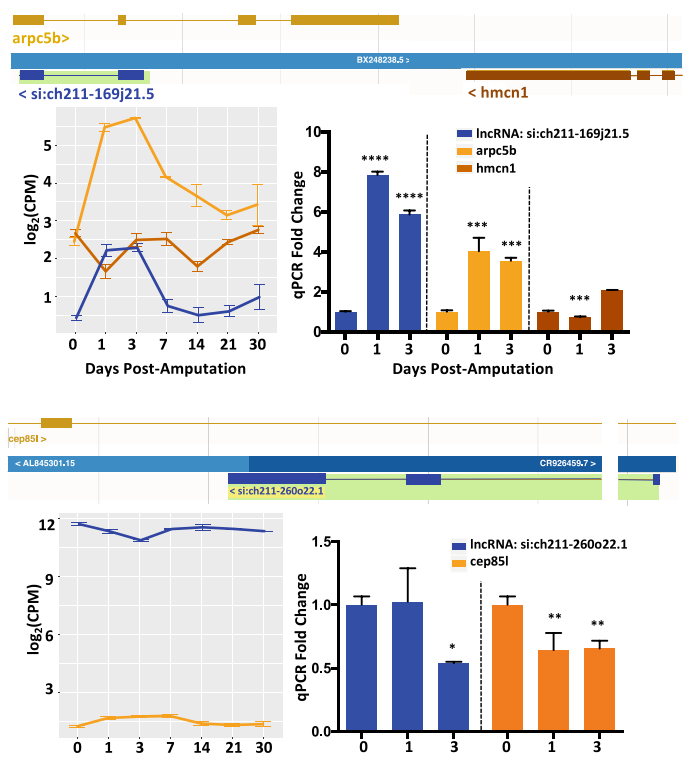
Days Post-Amputation
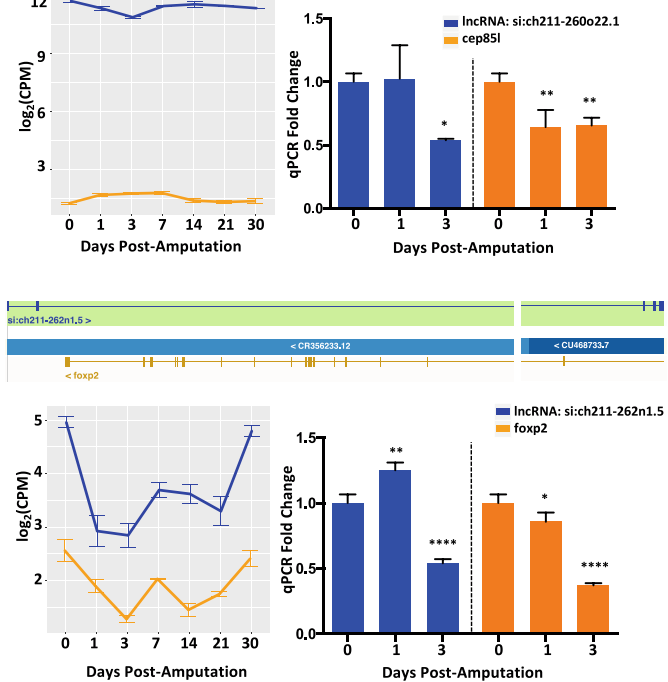

b
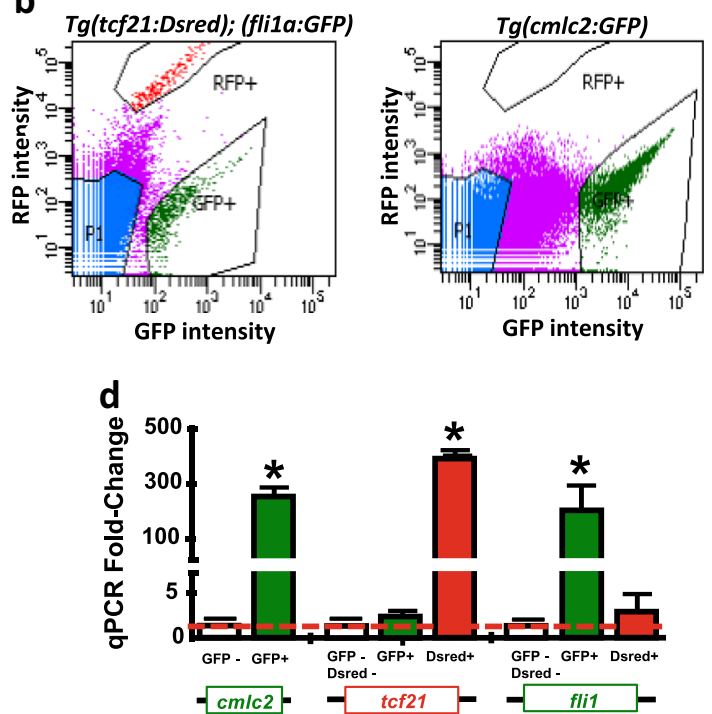

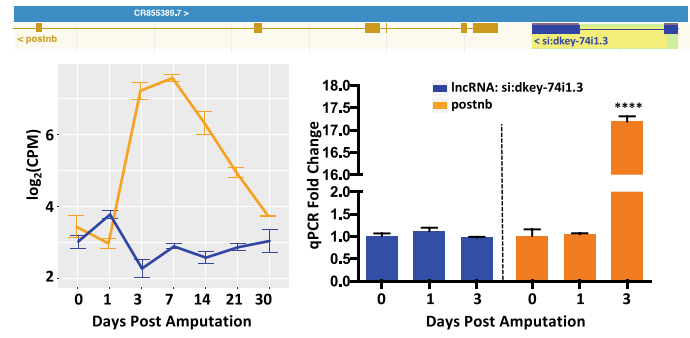

$10 \quad-1 \quad-1$
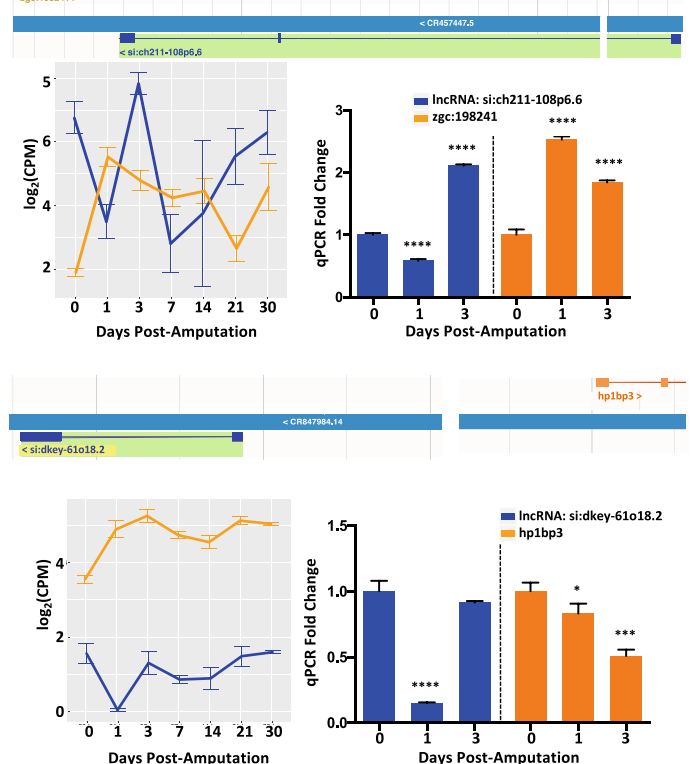

\begin{tabular}{|l|c|c|c|}
\hline \multicolumn{1}{|c|}{ Gene Symbol } & tcf21+ & cmlc2+ & fli1a+ \\
\hline IncRNA: si:ch211-169j21.5 & +++ & + & ++ \\
\hline arpc5b & +++ & + & ++ \\
\hline hmcn1 & ++ & + & +++ \\
\hline IncRNA: si:ch211-108p6.6 & $\mathrm{ND}$ & $\mathrm{ND}$ & $\mathrm{ND}$ \\
\hline zgc:198241 & +++ & + & +++ \\
\hline IncRNA: si:ch211-262n1.5 & +++ & + & ++ \\
\hline foxp2 & ++ & + & +++ \\
\hline IncRNA: si:dkey-74l1.3 & + & +++ & ++ \\
\hline postnb & +++ & + & ++ \\
\hline IncRNA: si:ch211-260o22.1 & +++ & + & ++ \\
\hline cep85I & +++ & + & +++ \\
\hline IncRNA: si:dkey-61018.2 & + & $\mathrm{ND}$ & +++ \\
\hline hp1bp3 & +++ & + & +++ \\
\hline
\end{tabular}

Fig. 4 Identification of known IncRNAs that were differentially expressed during early stages of zebrafish heart regeneration. a Schematic showing relative genomic location of IncRNAs and corresponding protein-coding genes from Ensembl, gene expression levels from RNA-Seq in $0,1,3,7,14,21$, and $30 \mathrm{dpa}$, and qPCR validation of expression in 0, 1, and $3 \mathrm{dpa}$ regenerating ventricles. Error bars indicate standard error of the mean (s.e.m). b Flow cytometry plots showing gating of epicardial (Tg(tcf21:Dsred)), endocardial (Tg(fli1a:GFP)), and cardiomyocyte (Tg (cm/c2:GFP)) cells from 3 dpa dissociated ventricles. c Relative expression of IncRNAs and antisense or adjacent protein-coding gene in tcf21+, $\mathrm{cmlc2}+$, and fli1+ cells at $3 \mathrm{dpa}$ by qPCR studies (ND not detected, $(+)$ low expression, $(++)$ 1.5-2.0-fold increase, $(+++) 2.0$ or higher-fold increase in expression). Error bars indicate s.e.m 

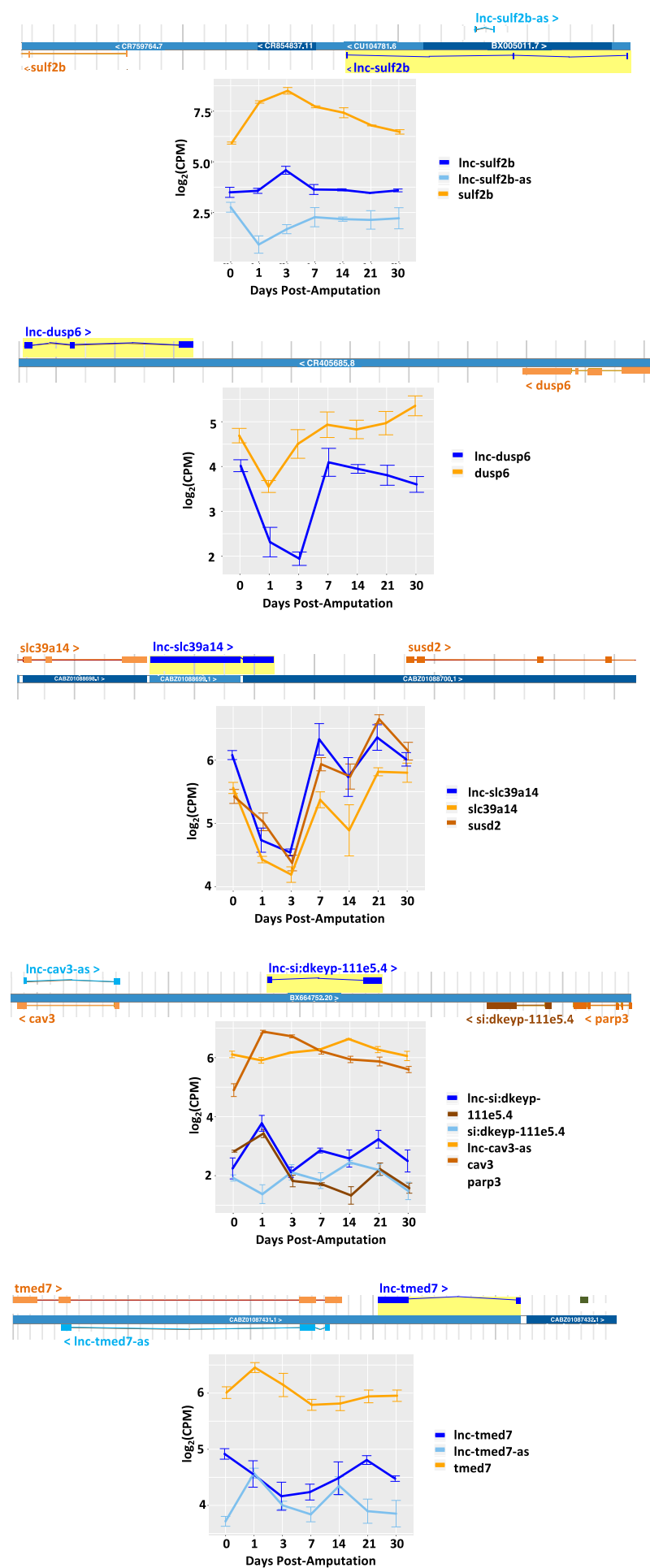

Fig. 5 Unique IncRNAs and associated target genes during zebrafish and mouse heart regeneration. Expression of select zebrafish novel IncRNAs adjacent to protein-coding genes and corresponding protein-coding genes that were differentially expressed at 1 and/ or 3 dpa relative to $0 \mathrm{dpa}$. Error bars indicate standard error of the mean

parp3 was proximal to poly(ADP-ribose) polymerase 3 (parp3). The mouse homolog, parp3, has been associated with cell cycle progression through DNA break repair and histone modification. ${ }^{76}$ Lnc-parp3 was also distal to caveolin 3 (cav3), a component of myocyte caveolae involved in natriuretic peptide signaling and cardiac hypertrophy in a mouse overexpression model, ${ }^{77}$ but cav3 was not differentially expressed. Finally, Inc-tmed7 was distal to transmembrane p24 trafficking protein 7 (tmed7), and proximal to mir7a-3, but mature Mir-7-P4 was not differentially expressed (Fig. $5)$. The proximity of these five putative novel IncRNAs to differentially expressed protein-coding genes make them strong candidate regulators of heart regeneration.

Comparative analysis of zebrafish and neonatal mouse heart regeneration

The ability to perform cross-species comparisons is necessary to identify a conserved regulatory circuit for regeneration of specific tissues. In contrast to other regeneration databases, RegenDbase represents orthologous genes, thereby enabling comparisons among organisms. Here, we compared a previously published RNA-Seq dataset from resected neonatal mouse hearts ${ }^{78}$ with our own profiling data from zebrafish hearts at comparable time points during regeneration. Neonatal murine hearts have been shown to fully regenerate missing heart muscle in a manner analogous to the adult zebrafish heart. ${ }^{79}$ For the neonatal mouse study, we used the expression data from the in vivo study so that we could directly compare regeneration in both models. We reanalyzed the RNA-Seq data from this study and found 215 differentially expressed genes from the $1 \mathrm{dpa}$ sham vs. $1 \mathrm{dpa}$ resection comparison and the 29 genes from the $7 \mathrm{dpa}$ sham vs. $7 \mathrm{dpa}$ resection comparison (Supplementary Table S12). A total of 68 ortholog groups were differentially expressed in at least one contrast in both organisms (Supplementary Table S13). A subset of those ortholog groups that are involved with several biological processes including the immune response, ion transport along with TGF $\beta, N F K B$, and integrin signaling (Supplementary Table S14). NFKB signaling has recently been demonstrated to be required for zebrafish heart regeneration. ${ }^{80}$ In that study, nfkbiaa was shown to be highly upregulated during regeneration. Two of the genes, mpeg 1 and marco, are associated with macrophages suggesting a common role of the immune system in regeneration, consistent with studies in the neonatal mouse. ${ }^{78}$ Six of these genes, bub1, casc5, cenpe, cenph, nuf2, and spc25, are involved in regulating cell cycling. These genes interact during the spindle assembly checkpoint during cell division. ${ }^{81}$

Looking beyond in vivo comparisons in the neonatal mouse study, we also examined the expression of il13 which has been shown to induce cardiomyocyte proliferation in vitro by regulating proliferation through Stat3 and Stat6. ${ }^{78}$ We observed that zebrafish il13 was upregulated by 11.14 -fold at $3 \mathrm{dpa}$, and stat 3 was upregulated by 1.74 -fold at $1 \mathrm{dpa}$ (Supplementary Table S12). Taken together, the striking similarity between zebrafish and mouse heart regeneration suggest common mechanisms and indicate zebrafish studies are highly informative for understanding mammalian tissue repair and regeneration.

To determine the interaction between IncRNAs with heart regeneration genes, we conducted a String analysis integrating differently regulated IncRNAs from the early stages of adult zebrafish and neonatal mouse heart regeneration. In the neonatal mouse heart, 12 IncRNAs were differentially expressed at $1 \mathrm{dpa}$ and two IncRNAs were differentially expressed at $7 \mathrm{dpa}$ (Fig. 6). Three of these 12 IncRNAs are antisense to protein-coding genes. Lnc-Gm15419 expression is upregulated and oriented antisense to the extracellular matrix gene, collagen $4 a 2$ (Col4a2). Likewise, LncGm26794 is also upregulated, but positioned antisense to the potassium channel, Kcnj3. A third IncRNA, Inc-Gm12167, was downregulated and is antisense to interleukin 2 inducible T-cell kinase, Itk. These interaction analyses suggest that a subset of IncRNA-adjacent mRNA units provides an additional layer of regulatory control on expression of conserved heart regeneration genes (Fig. 6). LncRNAs are a relatively new group of gene expression regulators and defining their functional roles during 


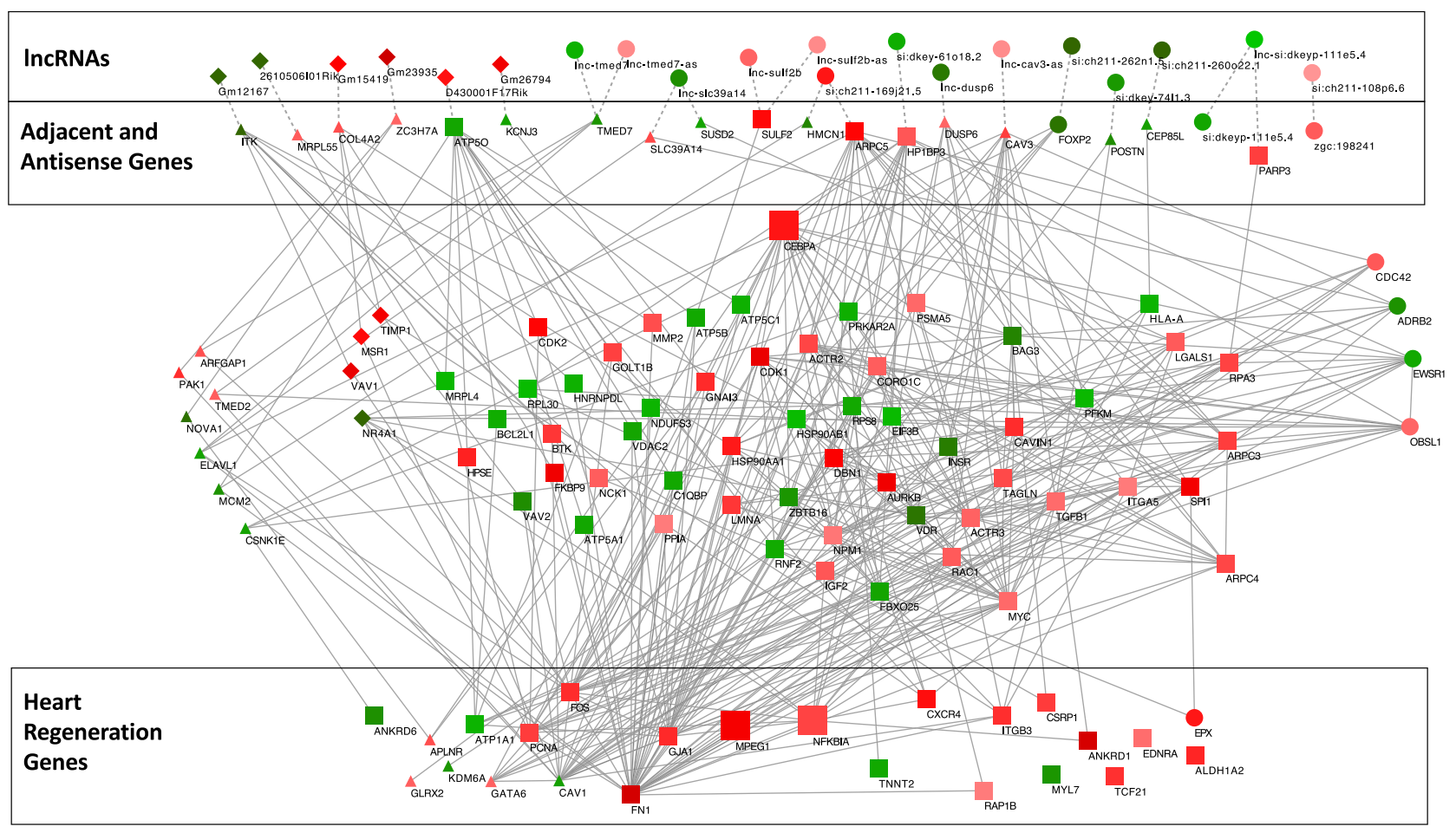

$\square$ Protein-coding orthologs expressed in zebrafish $(F D R<0.05)$ and mouse $(F D R<0.05)$
$\square$ Protein-coding orthologs expressed in zebrafish $(F D R<0.05)$ and mouse $(F D R>0.05)$
$\triangle$ Protein-coding orthologs expressed in mouse $(F D R<0.05)$ and zebrafish $(F D R>0.05)$

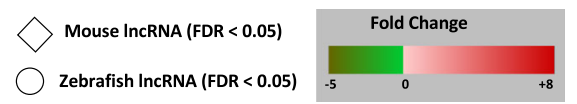

Fig. 6 Comparative model of IncRNA-regulation of heart regeneration. Known and novel zebrafish IncRNAs adjacent or antisense to proteincoding genes (top) were connected to a set of previously characterized genes required for heart regeneration in the zebrafish (bottom). Gene regulatory and/or protein-protein interactions from Ingenuity Pathways Analysis were used to find a minimal set of homologous genes that connect the adjacent/antisense genes to the regeneration genes. Genes differentially expressed in the zebrafish (0 vs. $3 \mathrm{dpa})$ and/or mouse ( 1 day sham vs. resection) are shown using different shapes and colored by fold change from the zebrafish or mouse (see legend)

heart regeneration in both regeneration competent and incompetent research systems is a critical next step toward understanding the regulatory complexity of heart regeneration.

\section{DISCUSSION}

We designed and built RegenDbase to facilitate the comparison of diverse regenerative models with a unique focus on noncoding RNAs, critical regulators of regeneration signaling pathways at multiple levels of gene expression. These comparisons may be performed among models with high regenerative capacity or between models with varying capacity. This resource represents gene expression datasets from multiple model systems relevant to regeneration. Our large RNA-sequencing study of zebrafish heart regeneration presented in this study serves as a foundation for comparative analyses to identify noncoding and protein encoding transcripts. The RegenDbase web user interface facilitates the rapid identification of genes differentially expressed within one experiment and across multiple experiments. These comparisons may also be conducted within or across organisms.

Previously, several developmental pathways have been shown to be reactivated following injury to orchestrate complex tissue regeneration. ${ }^{73,82-84}$ Protein-coding genes that make up developmental pathways are highly conserved across vertebrates and are part of the genetic arsenal that coordinate animal development. ${ }^{85}$ Yet, despite the evolutionary conservation of these circuits, the capacity to regenerate tissues is highly variable, suggesting additional layers of regulatory control of gene activation are key to inducing regeneration circuits.
Noncoding RNAs provide two additional layers of regulatory complexity that determine both spatial and relative intensity of developmental pathway activation (Supplementary Fig. S5). First, IncRNAs function in the nucleus to epigenetically regulate target genes that can positively or negatively influence gene expression. Second, noncoding RNAs also can function in the cytoplasm to regulate gene expression post-transcriptionally. In this second layer of genetic complexity, miRNAs negatively regulate target mRNAs, and IncRNAs positively and negatively regulate target gene expression.

RegenDbase facilitates the understanding of miRNA regulation by identifying sets of differentially expressed genes that are predicted to be targeted by miRNAs. As multiple miRNAs can regulate the same gene in a combinatorial fashion, RegenDbase also enables miRNA target searches to identify cases where up to three different miRNAs target the same gene. Using RegenDbase, we have demonstrated how to identify aspects of heart regeneration that are unique to the zebrafish and are conserved with neonatal mouse heart regeneration. The utility of RegenDbase will grow as more datasets from more models are added.

In contrast to miRNAs, IncRNAs are not highly conserved. It is interesting to speculate that a common set of IncRNAs may control common regeneration pathways for multiple tissues, or that different sets of noncoding RNAs are used to modulate regenerative signaling cascades for individual tissues. Further studies of noncoding RNAs in vertebrates with varying regenerative capacities will help define their roles and test the extent to which they do regulate regenerative capacity. Nonetheless, the 
newly identified noncoding RNAs provide new insight into the complexity of gene regulatory networks that govern regeneration.

RegenDbase has multiple additional search functions that support the development of novel hypotheses for regenerative biology (Supplementary Fig. S1). In this work, we have primarily focused on finding genes that were commonly differentially expressed between zebrafish and mouse. However, RegenDbase supports analysis of genes that are not commonly differentially expressed. For example, the regenerative potential of the neonatal mouse heart is reduced after 7 days. Genes that modulate regeneration may be identified through the comparison of genes that change during postnatal development and those associated with regeneration. In a similar fashion, comparisons of wound healing across organisms with high regenerative capacities to those with low capacities may identify genes that modulate these capacities. While this current release of RegenDbase is focused on zebrafish, axolotl, and mouse data, it is noteworthy to mention that we have established pipelines to integrate future datasets from $C$. elegans, human tissues, and other model organisms to further refine regenerative circuits through comparative analyses.

Additionally, search functionality and more datasets will enhance the system. Studies of inhibited or enhanced regeneration provide valuable insight into genetic mechanisms. Additional data on genomic regulatory features, such as chromatin state and methylation patterns, provide new opportunities to understand these mechanisms. Improvements in single-cell technologies will make it easier to study the contributions of different cell types on regeneration. We also plan to enhance comparative analysis capabilities to facilitate meta-analysis across experiments conducted using different technologies with varying sensitivity and representation of genes. When these experiments are conducted, resources like RegenDbase are primed to integrate, search, and visualize these data to form testable hypotheses for functional studies.

\section{METHODS}

Data collection, relational database, and web interface

Published datasets were loaded in the relational database and checked for any errors related to gene annotation. RegenDbase integrates datasets from 15 experiments and uses 24 external data resources that provide lists of genes and annotations of those genes (see https://regendbase.org/data for list). These resources include genes and transcripts from Ensembl (version 86) and miRBase (version 21), orthologs from OrthoDB (version 9), proteins from UniProt and annotations from the GO and NCBI BioSystems for pathways. miRNAs from miRBase and $3 p$ UTRs from Ensembl were used to make miRNA-binding sites predictions. These data were loaded into the relational database where we have implemented a robust data model (Supplementary Fig. S1). We have designed the system to update these data on a regular basis to take advantage of the latest annotation of genes and genomes.

Central to the RegenDbase data model are RNA segments that represent transcripts and microarray probes. These RNA segments were used to represent genes, assign expression data, and represent miRNA target predictions. Genes may have one or more RNA segments that were assigned any number of annotations. Representing homologous genes, such as orthologs, requires relating genes and their RNA segments to one another across organisms. For protein-coding genes, we represented groups of orthologous genes from OrthoDB (version 9). For axolotl, we first mapped of microarray probe set target sequences to an axolotl transcriptome assembly ${ }^{14}$ using NCBI BLAST blastn to return the first alignment with an expectation value less than $1.0 \times 10^{-5}$. The transcriptome contig that best represented the target sequence was then aligned to the human UniProt protein sequences using NCBI BLAST blastx. The first UniProt alignment returned by BLAST with an expectation value less than $1.0 \times 10^{-5}$ was used to assign to the microarray probe set to the axolotl gene record based on human UniProt mapping to the OrthoDB homolog group. For orthologous miRNAs, we used miRBase (version 21) mapping of miRNAs to RFAM miRNA families. Gene expression data were represented in the data model as experiments, with samples organized into sample groups. Normalized expression values were assigned to RNA segments for each sample. Precomputed comparisons of RNA segment expression between sample groups from the analysis pipelines were represented as fold change and adjusted $p$-values. All these data can then be quickly searched using the web interface. RegenDbase was implemented using Java and the PostgreSQL relational database management system (Supplementary Fig. S1). Source code was maintained using Subversion (http://subversion.apache.org).

\section{Animal husbandry and tissue collection}

All animal procedures were performed, with IACUC approval, in the Animal Core Facility at MDI Biological Laboratory. Female and male adult wild-type Ekkwill (EK) and transgenic zebrafish of 9-12 months of age were anesthetized by immersion in 1:1000 dilution of 2-phenoxyethanol and subjected to ventricular resection. Approximately $20 \%$ of the apex was removed, as previously described., ${ }^{5,12}$ Whole ventricles were collected at 0 , $1,3,7,14,21$, and 30 days post-amputation (dpa) for RNA extraction.

\section{RNA preparation and sequencing}

Total RNA samples were extracted from triplicate tissue samples using the Zymo Direct-zol RNA microprep kit (Zymo Research Corp., Irvine, CA). Each replicate consisted of 4-6 ventricles. Indexed small RNA libraries and strand-specific polyA + selected mRNA libraries were prepared and pairedend sequenced on an Illumina HiSeq2500 at the HudsonAlpha Institute for Biotechnology following the manufacturer's protocols. Sequence data are available in the Gene Expression Omnibus (GSE106884) and Short Read Archive (SRP124960).

\section{FACS and gene expression}

Resected transgenic adult zebrafish ventricles were extracted and dissociated in accordance to previously described methods. ${ }^{86}$ Isolation of fluorescently marked cells from $T g(t c f 21: D s r e d), T g(\mathrm{cmlc2}: G F P)$, and $T g$ (fli1a: GFP) was performed on a FACSAria II instrument (BD Biosciences) at the Jackson Laboratory (Bar Harbor, ME). Cells were sorted directly into Trizol LS and total RNA was isolated using Zymo Direct-zol RNA microprep kit, as suggested by the manufacturer (Zymo Research Corp., Irvine, CA). Total RNA was amplified with the Nugen Ovation Pico WTA System and CDNA was synthesized using NEB ProtoScript II First Strand CDNA kit, in accordance to the manufacturer's protocol (NEB, Ipswich, MA). Real-time qPCR expression studies were performed with SYBR Green (Agilent) detection and specific primers for each gene (Supplementary Table S15).

\section{Sequence annotation and RNA-Seq data analysis}

Following sequence read quality control diagnostic analyses using FastQC version 0.11 .2 (http://www.bioinformatics.babraham.ac.uk/projects/fastqc/ ), small RNA-Seq reads were adapter clipped and mapped to annotated zebrafish miRNAs from miRGeneDB version 1.1 (ref. ${ }^{87}$ ) using miRExpress version 2.0. ${ }^{88}$ Sequence tag counts for annotated mature miRNAs from miRGeneDB were analyzed using the $R$ statistical computing environment (http://r-project.org) version 3.2.1 and R/edgeR version 3.2.1. ${ }^{89}$ mRNA-Seq read data were also subjected to quality control diagnostic analyses using FastQC. Reads were trimmed using Trimmomatic version $0.32 .{ }^{90}$ Trimmed paired-end reads were aligned to the Ensembl annotated transcriptome (version 79) ${ }^{91}$ using RSEM version 1.2.25 (ref. ${ }^{92}$ ) and Bowtie 1.1.2. ${ }^{93}$ Read counts expressed as transcripts per million were analyzed using R/edgeR. All heatmaps were generated using the R/ComplexHeatmap version 1.0.0 package. GO enrichment analyses were performed using GOrill ${ }^{50}$ where all genes expressed at each time point were used as background. Zebrafish genes annotated to specific GO terms were obtained using AmiGO. ${ }^{23}$ miRNA-binding sites were predicted using miRanda version $3.3 a^{94}$ using annotated 3p UTRs from Ensembl version 79 and mature miRNA sequences from miRGeneDB version 1.1. Novel IncRNAs were identified by aligning the RNA-Seq reads to the zebrafish genome using HISAT2 version $2.0 .5,95$ generating gene models using StringTie version $1.3 .1 c^{71}$ comparing transcripts to Ensembl version 79 annotation using gffcompare. Novel genes were filtered for repetitive sequences using RepeatMasker and coding potential by CPAT version 1.2.1. ${ }^{96}$

\section{Disclaimer}

The contents of this paper are solely the responsibility of the authors and do not necessarily represent the official views of the NIH or other funding agencies 
Data availability

All expression analysis data have been deposited at the NCBI Gene Expression Omnibus (GSE106884) and the NCBI Short Read Archive (SRP124960).

\section{Code availability}

The web interface to RegenDbase is freely accessible at https:// regendbase.org.

\section{ACKNOWLEDGEMENTS}

We thank Dr. Ken Poss from Duke Medical Center for generously providing us with zebrafish $T g$ (tcf21:Dsred), $T g$ (cm/c2:GFP), and Tg(fli1a:GFP) transgenic strains. We thank Ari Dehn, Karlee Markovich, and Anne Yu for excellent zebrafish care at MDI Biological Laboratory. We are grateful to MaryLynn FitzSimons in the Yin lab and Dr. William Schott at the Jackson Laboratory Flow Cytometry Scientific Services for their guidance and assistance with cell sorting experiments. This work was supported by Center of Biomedical Research Excellence (COBRE) grant P20 GM104318 and by Institutional Development Award (IDeA) grant P20 GM103423 from the National Institute of General Medical Sciences. Additional support was provided to V.P.Y. by a Scott A. MacKenzie Foundation Award (17003) and an American Heart Association Scientist Development Grant (11SDG7210045).

\section{AUTHOR CONTRIBUTIONS}

B.L.K. and V.P.Y. designed the experiment, analyzed the data, and wrote the manuscript. B.L.K. and M.C.R. designed the data model and M.C.R. wrote the software for RegenDbase. B.L.K. and G.A.S. established the pipeline that identified putative IncRNAs. A.M.S. and C.A.D. performed heart surgeries, gene expression and FACS experiments, and analyzed the data. C.A.D. illustrated the images in Figure 1A. All authors have read and approved the final version of the manuscript.

\section{ADDITIONAL INFORMATION}

Supplementary information accompanies the paper on the npj Regenerative Medicine website (https://doi.org/10.1038/s41536-018-0049-0).

Competing interests: The authors declare no competing interests.

Publisher's note: Springer Nature remains neutral with regard to jurisdictional claims in published maps and institutional affiliations.

\section{REFERENCES}

1. Neves, J., Sousa-Victor, P. \& Jasper, H. Rejuvenating strategies for stem cell-based therapies in aging. Cell Stem Cell 20, 161-175 (2017).

2. Chandel, N. S., Jasper, H., Ho, T. T. \& Passegue, E. Metabolic regulation of stem cell function in tissue homeostasis and organismal ageing. Nat. Cell Biol. 18, 823-832 (2016).

3. Yao, B. et al. Age-associated changes in regenerative capabilities of mesenchymal stem cell: impact on chronic wounds repair. Int. Wound J. 13, 1252-1259 (2016).

4. Loperfido, M., Steele-Stallard, H. B., Tedesco, F. S. \& VandenDriessche, T. Pluripotent stem cells for gene therapy of degenerative muscle diseases. Curr. Gene Ther. 15, 364-380 (2015).

5. Poss, K. D., Wilson, L. G. \& Keating, M. T. Heart regeneration in zebrafish. Science 298, 2188-2190 (2002).

6. Becker, T., Wullimann, M. F., Becker, C. G., Bernhardt, R. R. \& Schachner, M. Axonal regrowth after spinal cord transection in adult zebrafish. J. Comp. Neurol. 377, 577-595 (1997).

7. White, J. A., Boffa, M. B., Jones, B. \& Petkovich, M. A zebrafish retinoic acid receptor expressed in the regenerating caudal fin. Development 120, 1861-1872 (1994).

8. Stocum, D. L. The urodele limb regeneration blastema. Determination and organization of the morphogenetic field. Differ. Res. Biol. Divers. 27, 13-28 (1984).

9. Kumar, A., Godwin, J. W., Gates, P. B., Garza-Garcia, A. A. \& Brockes, J. P. Molecular basis for the nerve dependence of limb regeneration in an adult vertebrate. Science 318, 772-777 (2007).

10. Yin, V. P. et al. Fgf-dependent depletion of microRNA-133 promotes appendage regeneration in zebrafish. Genes Dev. 22, 728-733 (2008).

11. Thatcher, E. J., Paydar, I., Anderson, K. K. \& Patton, J. G. Regulation of zebrafish fin regeneration by microRNAs. Proc. Natl. Acad. Sci. USA 105, 18384-18389 (2008).
12. Yin, V. P., Lepilina, A., Smith, A. \& Poss, K. D. Regulation of zebrafish heart regeneration by miR-133. Dev. Biol. 365, 319-327 (2012).

13. Beauchemin, M., Smith, A. \& Yin, V. P. Dynamic microRNA-101a and Fosab expression controls zebrafish heart regeneration. Development 142, 4026-4037 (2015).

14. King, B. L. \& Yin, V. P. A conserved microRNA regulatory circuit is differentially controlled during limb/appendage regeneration. PLOS ONE 11, e0157106 (2016).

15. Lobo, D., Feldman, E. B., Shah, M., Malone, T. J. \& Levin, M. A bioinformatics expert system linking functional data to anatomical outcomes in limb regeneration. Regeneration 1, 37-56 (2014).

16. Zhao, M., Rotgans, B., Wang, T. \& Cummins, S. F. REGene: a literature-based knowledgebase of animal regeneration that bridge tissue regeneration and cancer. Sci. Rep. 6, 23167 (2016).

17. Clough, E. \& Barrett, T. The Gene Expression Omnibus Database. Methods Mol. Biol. 1418, 93-110 (2016).

18. Petryszak, R. et al. Expression Atlas update-an integrated database of gene and protein expression in humans, animals and plants. Nucleic Acids Res. 44, D746-D752 (2016).

19. Consortium, G. T. The Genotype-Tissue Expression (GTEx) project. Nat. Genet. 45, 580-585 (2013)

20. Cho, S. et al. MiRGatorv3.0: a microRNA portal for deep sequencing, expression profiling and mRNA targeting. Nucleic Acids Res. 41, D252-D257 (2013).

21. Yates, A. et al. Ensembl 2016. Nucleic Acids Res. 44, D710-D716 (2016).

22. Kozomara, A. \& Griffiths-Jones, S. miRBase: annotating high confidence microRNAs using deep sequencing data. Nucleic Acids Res. 42, D68-D73 (2014).

23. Carbon, S. et al. AmiGO: online access to ontology and annotation data. Bioinformatics 25, 288-289 (2009).

24. Geer, L. Y. et al. The NCBI BioSystems database. Nucleic Acids Res. 38, D492-D496 (2010).

25. Kriventseva, E. V. et al. OrthoDBv8: update of the hierarchical catalog of orthologs and the underlying free software. Nucleic Acids Res. 43, D250-D256 (2015).

26. Kurian, L. et al. Identification of novel long noncoding RNAs underlying vertebrate cardiovascular development. Circulation 131, 1278-1290 (2015).

27. Walker, M. B., King, B. L. \& Paigen, K. Clusters of ancestrally related genes that show paralogy in whole or in part are a major feature of the genomes of humans and other species. PLOS ONE 7, e35274 (2012).

28. Itou, J. et al. Migration of cardiomyocytes is essential for heart regeneration in zebrafish. Development 139, 4133-4142 (2012).

29. Gemberling, M., Bailey, T. J., Hyde, D. R. \& Poss, K. D. The zebrafish as a model for complex tissue regeneration. Trends Genet. 29, 611-620 (2013).

30. Chen, J. F. et al. The role of microRNA-1 and microRNA-133 in skeletal muscle proliferation and differentiation. Nat. Genet. 38, 228-233 (2006).

31. Giraldez, A. J. et al. MicroRNAs regulate brain morphogenesis in zebrafish. Science 308, 833-838 (2005).

32. Bhaumik, D. et al. Expression of microRNA-146 suppresses NF-kappaB activity with reduction of metastatic potential in breast cancer cells. Oncogene 27, 5643-5647 (2008).

33. Holman, E. C., Campbell, L. J., Hines, J. \& Crews, C. M. Microarray analysis of microRNA expression during axolotl limb regeneration. PLOS ONE 7, e41804 (2012).

34. Meng, F. et al. MicroRNA-21 regulates expression of the PTEN tumor suppressor gene in human hepatocellular cancer. Gastroenterology 133, 647-658 (2007).

35. Ryan, B. M. microRNAs in cancer susceptibility. Adv. Cancer Res. 135, 151-171 (2017).

36. Drusco, A. \& Croce, C. M. MicroRNAs and cancer: a long story for short RNAs. Adv. Cancer Res. 135, 1-24 (2017).

37. Chatterjee, N., Rana, S., Espinosa-Diez, C. \& Anand, S. MicroRNAs in cancer: challenges and opportunities in early detection, disease monitoring, and therapeutic agents. Curr. Pathobiol. Rep. 5, 35-42 (2017).

38. Kikuchi, K. et al. Retinoic acid production by endocardium and epicardium is an injury response essential for zebrafish heart regeneration. Dev. Cell 20, 397-404 (2011).

39. Wang, J., Karra, R., Dickson, A. L. \& Poss, K. D. Fibronectin is deposited by injuryactivated epicardial cells and is necessary for zebrafish heart regeneration. Dev. Biol. 382, 427-435 (2013).

40. Gonzalez-Rosa, J. M., Martin, V., Peralta, M., Torres, M. \& Mercader, N. Extensive scar formation and regression during heart regeneration after cryoinjury in zebrafish. Development 138, 1663-1674 (2011).

41. Kikuchi, K. et al. tcf21 + epicardial cells adopt non-myocardial fates during zebrafish heart development and regeneration. Development 138, 2895-2902 (2011).

42. Yelon, D., Horne, S. A. \& Stainier, D. Y. Restricted expression of cardiac myosin genes reveals regulated aspects of heart tube assembly in zebrafish. Dev. Biol. 214, 23-37 (1999). 
43. Lackner, S., Schwendinger-Schreck, J., Julich, D. \& Holley, S. A. Segmental assembly of fibronectin matrix requires rap1b and integrinalpha5. Dev. Dyn. 242, 122-131 (2013).

44. Dong, W. et al. Suppression of Rap1 impairs cardiac myofibrils and conduction system in zebrafish. PLOS ONE 7, e50960 (2012).

45. Miyasaka, K. Y., Kida, Y. S., Sato, T., Minami, M. \& Ogura, T. Csrp1 regulates dynamic cell movements of the mesendoderm and cardiac mesoderm through interactions with Dishevelled and Diversin. Proc. Natl. Acad. Sci. USA 104, 11274-11279 (2007).

46. Becker, J. R. et al. Differential activation of natriuretic peptide receptors modulates cardiomyocyte proliferation during development. Development 141, 335-345 (2014).

47. Sato, M., Tsai, H. J. \& Yost, H. J. Semaphorin3D regulates invasion of cardiac neural crest cells into the primary heart field. Dev. Biol. 298, 12-21 (2006).

48. Li, L., Miano, J. M., Cserjesi, P. \& Olson, E. N. SM22 alpha, a marker of adult smooth muscle, is expressed in multiple myogenic lineages during embryogenesis. Circ. Res. 78, 188-195 (1996).

49. Xiong, B. et al. Tob1 controls dorsal development of zebrafish embryos by antagonizing maternal beta-catenin transcriptional activity. Dev. Cell 11, 225-238 (2006).

50. Eden, E., Navon, R., Steinfeld, I., Lipson, D. \& Yakhini, Z. GOrilla: a tool for discovery and visualization of enriched $\mathrm{GO}$ terms in ranked gene lists. BMC Bioinformatics 10, 48 (2009)

51. Paffett-Lugassy, N. et al. Functional conservation of erythropoietin signaling in zebrafish. Blood 110, 2718-2726 (2007).

52. Kardon, J. R. et al. Mitochondrial ClpX activates a key enzyme for heme biosynthesis and erythropoiesis. Cell 161, 858-867 (2015).

53. Ransom, D. G. et al. Characterization of zebrafish mutants with defects in embryonic hematopoiesis. Development 123, 311-319 (1996).

54. Takahashi, K. \& Yamanaka, S. Induction of pluripotent stem cells from mouse embryonic and adult fibroblast cultures by defined factors. Cell 126, 663-676 (2006).

55. Kotkamp, K., Mossner, R., Allen, A., Onichtchouk, D. \& Driever, W. A Pou5f1/Oct4 dependent KIf2a, KIf2b, and Klf17 regulatory sub-network contributes to EVL and ectoderm development during zebrafish embryogenesis. Dev. Biol. 385, 433-447 (2014).

56. Kikuchi, K. Advances in understanding the mechanism of zebrafish heart regeneration. Stem Cell Res. 13, 542-555 (2014).

57. Lim, L. P. et al. Microarray analysis shows that some microRNAs downregulate large numbers of target mRNAs. Nature 433, 769-773 (2005).

58. Huang, G. N. et al. C/EBP transcription factors mediate epicardial activation during heart development and injury. Science 338, 1599-1603 (2012).

59. Batista, P. J. \& Chang, H. Y. Long noncoding RNAs: cellular address codes in development and disease. Cell 152, 1298-1307 (2013).

60. Batista, P. J. \& Chang, H. Y. Cytotopic localization by long noncoding RNAs. Curr Opin. Cell Biol. 25, 195-199 (2013).

61. Chen, Y. M. et al. Identification of differentially expressed IncRNAs involved in transient regeneration of the neonatal $\mathrm{C} 5 \mathrm{BBL} / 6 \mathrm{~J}$ mouse heart by next-generation high-throughput RNA sequencing. Oncotarget 8, 28052-28062 (2017).

62. Guttman, $M$. et al. lincRNAs act in the circuitry controlling pluripotency and differentiation. Nature 477, 295-300 (2011).

63. Gupta, R. A. et al. Long non-coding RNA HOTAIR reprograms chromatin state to promote cancer metastasis. Nature 464, 1071-1076 (2010).

64. Brunner, A. L. et al. Transcriptional profiling of long non-coding RNAs and novel transcribed regions across a diverse panel of archived human cancers. Genome Biol. 13, R75 (2012)

65. Bond, A. M. et al. Balanced gene regulation by an embryonic brain ncRNA is critical for adult hippocampal GABA circuitry. Nat. Neurosci. 12, 1020-1027 (2009).

66. Bertani, S., Sauer, S., Bolotin, E. \& Sauer, F. The noncoding RNA Mistral activates Hoxa6 and Hoxa7 expression and stem cell differentiation by recruiting MLL1 to chromatin. Mol. Cell 43, 1040-1046 (2011).

67. Chowdhury, A. et al. Expression of fibulin- 6 in failing hearts and its role for cardiac fibroblast migration. Cardiovasc. Res. 103, 509-520 (2014).

68. Winkelmann, N. et al. Recurrent CEP85L-PDGFRB fusion in patient with $t(5 ; 6)$ and imatinib-responsive myeloproliferative neoplasm with eosinophilia. Leuk. Lymphoma 54, 1527-1531 (2013).

69. Enard, W. et al. A humanized version of Foxp2 affects cortico-basal ganglia circuits in mice. Cell 137, 961-971 (2009).

70. Dutta, B. et al. Profiling of the chromatin-associated proteome identifies HP1BP3 as a novel regulator of cell cycle progression. Mol. Cell Proteomics (2014).

71. Pertea, M. et al. StringTie enables improved reconstruction of a transcriptome from RNA-seq reads. Nat. Biotechnol. 33, 290-295 (2015).
72. Lum, D. H., Tan, J., Rosen, S. D. \& Werb, Z. Gene trap disruption of the mouse heparan sulfate 6-O-endosulfatase gene, Sulf2. Mol. Cell Biol. 27, 678-688 (2007).

73. Stoick-Cooper, C. L. et al. Distinct Wnt signaling pathways have opposing roles in appendage regeneration. Development 134, 479-489 (2007).

74. Maillet, M. et al. DUSP6 (MKP3) null mice show enhanced ERK1/2 phosphorylation at baseline and increased myocyte proliferation in the heart affecting disease susceptibility. J. Biol. Chem. 283, 31246-31255 (2008).

75. Watson, A. P., Evans, R. L. \& Egland, K. A. Multiple functions of sushi domain containing 2 (SUSD2) in breast tumorigenesis. Mol. Cancer Res 11, 74-85 (2013).

76. Boehler, C. et al. Poly(ADP-ribose) polymerase 3 (PARP3), a newcomer in cellular response to DNA damage and mitotic progression. Proc. Natl. Acad. Sci. USA 108 2783-2788 (2011).

77. Horikawa, Y. T. et al. Cardiac-specific overexpression of caveolin-3 attenuates cardiac hypertrophy and increases natriuretic peptide expression and signaling. $J$. Am. Coll. Cardiol. 57, 2273-2283 (2011).

78. O'Meara, C. C. et al. Transcriptional reversion of cardiac myocyte fate during mammalian cardiac regeneration. Circ. Res. 116, 804-815 (2015).

79. Porrello, E. R. et al. Transient regenerative potential of the neonatal mouse heart Science 331, 1078-1080 (2011).

80. Karra, R., Knecht, A. K., Kikuchi, K. \& Poss, K. D. Myocardial NF-kappaB activation is essential for zebrafish heart regeneration. Proc. Natl. Acad. Sci. USA 112 13255-13260 (2015).

81. Hutchins, J. R. et al. Systematic analysis of human protein complexes identifies chromosome segregation proteins. Science 328, 593-599 (2010).

82. Tanaka, E. M. \& Reddien, P. W. The cellular basis for animal regeneration. Dev. Cell 21, 172-185 (2011)

83. Tzahor, E. \& Poss, K. D. Cardiac regeneration strategies: staying young at heart Science 356, 1035-1039 (2017).

84. Yokoyama, $\mathrm{H}$. et al. Different requirement for Wnt/beta-catenin signaling in limb regeneration of larval and adult Xenopus. PLOS ONE 6, e21721 (2011).

85. Chen, C. H. \& Poss, K. D. Regeneration genetics. Annu. Rev. Genet. (2017).

86. Cao, J. et al. Single epicardial cell transcriptome sequencing identifies Caveolin 1 as an essential factor in zebrafish heart regeneration. Development 143, 232-243 (2016).

87. Fromm, B. et al. A uniform system for the annotation of vertebrate microRNA genes and the evolution of the human microRNAome. Annu. Rev. Genet. 49, 213-242 (2015).

88. Wang, W. C. et al. miRExpress: analyzing high-throughput sequencing data for profiling microRNA expression. BMC Bioinformatics 10, 328 (2009).

89. Robinson, M. D., McCarthy, D. J. \& Smyth, G. K. edgeR: a Bioconductor package for differential expression analysis of digital gene expression data. Bioinformatics $\mathbf{2 6}$ 139-140 (2010).

90. Bolger, A. M., Lohse, M. \& Usadel, B. Trimmomatic: a flexible trimmer for Illumina sequence data. Bioinformatics 30, 2114-2120 (2014).

91. Flicek, P. et al. Ensembl 2014. Nucleic Acids Res. 42, D749-D755 (2014).

92. Li, B. \& Dewey, C. N. RSEM: accurate transcript quantification from RNA-Seq data with or without a reference genome. BMC Bioinformatcs 12, 323 (2011).

93. Langmead, B., Trapnell, C., Pop, M. \& Salzberg, S. L. Ultrafast and memory-efficient alignment of short DNA sequences to the human genome. Genome Biol. 10, R25 (2009).

94. Enright, A. J. et al. MicroRNA targets in Drosophila. Genome Biol. 5, R1 (2003).

95. Kim, D., Langmead, B. \& Salzberg, S. L. HISAT: a fast spliced aligner with low memory requirements. Nat. Methods 12, 357-360 (2015)

96. Wang, L. et al. CPAT: Coding-Potential Assessment Tool using an alignment-free logistic regression model. Nucleic Acids Res. 41, e74 (2013).

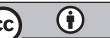

Open Access This article is licensed under a Creative Commons Attribution 4.0 International License, which permits use, sharing, adaptation, distribution and reproduction in any medium or format, as long as you give appropriate credit to the original author(s) and the source, provide a link to the Creative Commons license, and indicate if changes were made. The images or other third party material in this article are included in the article's Creative Commons license, unless indicated otherwise in a credit line to the material. If material is not included in the article's Creative Commons license and your intended use is not permitted by statutory regulation or exceeds the permitted use, you will need to obtain permission directly from the copyright holder. To view a copy of this license, visit http://creativecommons. org/licenses/by/4.0/.

(c) The Author(s) 2018 